\title{
Potential negative impacts and low effectiveness in the use of African annual killifish in the biocontrol of aquatic mosquito larvae in temporary water bodies
}

\author{
Martin Reichard ${ }^{1 *}$, Brian R Watters ${ }^{2}$, Rudolf H Wildekamp ${ }^{3}$, Rainer Sonnenberg ${ }^{4}$, Béla Nagy ${ }^{5}$, Matej Polač;ik ${ }^{6}$, \\ Stefano Valdesalici ${ }^{7}$, Alessandro Cellerino ${ }^{8}$, Barry J Cooper ${ }^{9}$, Holger Hengstler $^{10}$, John Rosenstock ${ }^{11}$, \\ lan Sainthouse ${ }^{12}$
}

\begin{abstract}
Commentary and discussion on a recent paper promoting the use of Nothobranchius guentheri, a small African annual fish from the Island of Zanzibar as a tool to control mosquito larvae in temporary bodies of freshwater throughout Africa is presented.

Arguments on major points; (1) expected low success of annual fish introductions, (2) low success of mosquito control in the field, (3) ecological threats, and (4) ethical issues are detailed.

Despite serious problems with mosquito-borne diseases in tropical Africa and elsewhere, we encourage responsible means of biological control of parasite vectors. We show that effectiveness of Nothobranchius translocations is low (the previous attempts failed), likelihood of effective mosquito larvae control under field condition is negligible and ecological threats from Nothobranchius translocations from within and outside the naturally occurring range are serious. We advocate against the proposed next step of the project, i.e. field trials in Tanzania.
\end{abstract}

\section{Letter}

Dear Editor

In a recent issue of Parasites \& Vectors, Matias and Adrias [1] report on their experiments on food preference of Nothobranchius guentheri, a small annual fish from Zanzibar Island (Tanzania), and conclude that the fish may become an ideal "tool to be employed in the eradication of diseases carried by mosquitoes through vector control, particularly in temporary bodies of freshwater". The authors describe previous suggestions to use Nothobranchius spp. for mosquito control [2-6] and expand on them to promote Nothobranchius guentheri as an ideal species to decrease mosquito larval densities in affected tropical areas. Matias and Adrias used seminatural experiments at the Poseidon Sciences field laboratory in the Philippines to show that $N$. guentheri

\footnotetext{
* Correspondence: reichard@ivb.cz

'Institute of Vertebrate Biology, Academy of Sciences of the Czech Republic, Květná 8, 60365 Brno, Czech Republic

Full list of author information is available at the end of the article
}

preferred to prey upon Culex quinquefasciatus larvae over chironomid larvae (Chironomus plumosus) and rotifers (Brachionus sp.). The authors showed that the presence of three fish per $\mathrm{m}^{2}$ of water surface was sufficient for the total eradication of C. quinquefasciatus larvae within four days. Finally, the authors suggested that $N$. guentheri can be conveniently transported in the form of diapausing embryos and then stocked in temporary water bodies that represent habitats for mosquito larvae, and conclude that they can effectively reduce mosquito larval populations [1].

We are well aware of the high risk of malaria and other mosquito-borne diseases in the tropics and we applaud any attempt to reduce the risk of contracting vector-borne diseases in those areas and elsewhere. At the same time, we think that the suggested approach has several important shortcomings, both from practical and ethical points of view. Our opinion is that the suggested practice would be largely ineffective and pose a significant threat to natural ecosystems. Our three major 
points (expected low success of annual fish introductions; low success of mosquito control in the field; and ecological threats) are detailed below. Some of us (RHW, BRW, BN) were in contact with the lead author of the study before its publication [1], commented on some aspects, and specifically mentioned some of the implications discussed in this response. Some of our concerns were addressed, but many were ignored. We question the overall practicality of the use of annual fishes as mosquito control in temporary water bodies and especially the introduction of $N$. guentheri or any other species of the genus to non-native areas inhabited by indigenous species of the same genus. We believe that the authors of the study should seriously consider modifications to their approach if the decision is made to proceed to the second step of their project, field trials in Tanzania [7].

\section{Low effectiveness of Nothobranchius fish introductions}

Nothobranchius fishes require very special habitat conditions. They cannot survive through more than one generation in pools that do not have the necessary habitat conditions, primarily an appropriate substrate for egg survival and development. Perhaps the most comprehensive account on habitat requirements of Nothobranchius is by Watters [8] who, based on field observations, experimental data and published studies, discusses the conditions that prevail in Nothobranchius habitats and the factors that control their distribution.

Nothobranchius fishes have been present in East Africa for many million years and during that time, geomorphological evolution of the landscape, with accompanying changes in drainage patterns, have been very effective in spreading Nothobranchius species to all sites that have the conditions necessary for their survival over the long term $[8,9]$. In other words, if Nothobranchius are capable of existing as a viable population in any particular seasonal pool then natural processes have ensured that they are already there. Brian Watters examined more than a thousand Nothobranchius habitats $[10,11]$, as well as pools that do not host Nothobranchius fishes. In all cases, the reasons for the presence or absence of Nothobranchius were primarily determined by the nature of the substrate. Without the particular type of substrate (alluvial vertisols) no eggs can survive the dry season.

During the course of an extensive project in the Kruger National Park (KNP) in South Africa, numerous sites in the northern part of the park, to which Nothobranchius had been translocated, were examined $[10,11]$. Two species of Nothobranchius (N. rachovii, N. orthonotus) occur naturally at only two localities in the park and, for conservation purposes, a decision was made in the mid-1970 $\mathrm{s}$ to translocate specimens of these two species to various seasonal pools in a different part of the park. These efforts were carried out during the period 1975-1985 and involved 10 different sites and 15 translocation events. The translocations involved large numbers of fish that were transported at considerable expense (by helicopter in many cases). None of these translocations were successful over even a relatively short term and only in a few cases did the fish survive through a full seasonal cycle. The investigations showed conclusively that the failure of these translocations was due primarily to an unsuitable substrate at the translocation sites that could not sustain the viability of eggs deposited therein by the fish. The only reason that in a few rare cases the translocated populations were able to survive through a seasonal cycle was because the translocations occurred during a particularly wet period. When normal conditions returned the populations became extinct [10].

Further, indirect evidence for the low success of Nothobranchius translocation comes from the original translocations by Vanderplank [12] who initiated introductions of $N$. taeniopygus from the vicinity of Old Shinyanga to other regions in Tanzania (Dodoma area), Uganda, Kenya and Swaziland. Numerous sampling efforts have been made in many of these areas over decades since these events, but no evidence for the survival of the introduced $N$. taeniopygus has ever been found [13].

The most important factor that makes the translocation of Nothobranchius fishes to new habitats pointless is that any seasonal pool within the overall range of distribution of Nothobranchius that is capable of sustaining a population of these fishes will already host one or more species of Nothobranchius. It makes no sense to introduce a new, non-native species (or population) into a pool that already hosts other species/populations of the same genus. Research carried out on the factors that control the distribution of Nothobranchius species over time $[9,14]$ provides evidence for this as well as the vast field observational experience of the authors who regularly conduct field studies of Nothobranchius fishes in the wild.

Tanzania, a potential target of suggested introductions [7], is rich in Nothobranchius fishes and they are present in almost every temporary body of water with suitable environmental conditions $[15,16]$. Unfortunately, it appears that Matias and Adrias have not made a serious effort to research this and to determine what the natural distribution and population density of these fishes are before suggesting a scheme that will have no greater effect on the mosquito population than existing populations of Nothobranchius already have.

In summary, introductions to sites presently lacking Nothobranchius fishes will very likely have the same negative results (i.e. no population establishment in new habitats) as previous attempts. 


\section{The effectiveness of eradication of mosquito larvae in the wild}

Most Nothobranchius species readily feed on mosquito larvae, including larval Culex spp. [15] and mosquito larvae are used as the most common diet for captive Nothobranchius [16]. This finding has been quantitatively confirmed by Matias and Adrias [1]. Studies on the diet of Nothobranchius in the wild are rare and Matias and Adrias cite a work which found undetermined mosquito larvae as main food item for a Nothobranchius species from Somalia. We suppose that the exact reference in the manuscript is incorrect as it suggests that the statement refers to findings from an experimental study concerning the resistance of egg chorion to external chemical damage [17], and we deduce that this information comes from a WHO (World Health Organization) report by Wildekamp [4] on $N$. microlepis or previous WHO assignment reports $[18,19]$ describing the diet of Nothobranchius patrizii (then determined as N. palmqvisti). However, Matias and Adrias selectively used only a part of the study that supports their results. The report by Wildekamp [4] indeed describes the frequent presence of mosquito larvae in $N$. microlepis stomachs. This report also contains data showing that $N$. microlepis had a clear preference for small planktonic crustaceans and their nauplii. The consumed mosquito larvae, mentioned in the report [4] were taken during experimental trials when mosquito larvae were the only food offered. Nothobranchius microlepis may be ecologically distinct from $N$. guentheri used by Matias and Adrias since it was discovered later that $N$. microlepis have specially adapted gill rakers to prey on small food items [20]. Mosquito larvae were frequently found in the diet of $N$. cyaneus $(=N$. jubbi) but they also frequently preyed on larger sized planktonic crustaceans and Coryxa nymphs (Hemiptera), and the conclusion that Nothobranchius have a preference for mosquito larvae is certainly not supported by [4] despite showing that mosquito larvae were frequently consumed (together with some other prey types) by some species.

Another recent study examined the diet of three sympatric species of Nothobranchius in southern Mozambique and found that mosquito larvae were extremely rare items in the diet of all three Nothobranchius species studied [21], while crustaceans (in N. furzeri and N. racho$v i i$, two species with body size and morphology comparable to $N$. guentheri) and coarse insect larvae such as Odonata, Ephemeroptera and Coleoptera (in $N$. orthonotus, a larger species) were the primary prey. We acknowledge the possibility that mosquito larvae may have already been eradicated if they constituted the preferred food and hence were not encountered during diet analysis, but the conservative interpretation is that mosquito larvae may not be as important component of the Nothobranchius diet in the wild, especially considering that the natural habitats of annual fishes and mosquito larvae are not identical.

Yet another pitfall with regard to the ability of Nothobranchius to eradicate mosquito larvae in the wild is that very substantial numbers of larvae would survive in the wide margins of natural pools overgrown by thick grass vegetation [14] in water too shallow for Nothobranchius fishes to reach. Mosquito larvae tend to be concentrated in the marginal zones of water bodies [22] and anopheline larvae typically reside among vegetation with limited motion and hence do not attract visual predators (such as Nothobranchius) [23]. This sedentary behaviour is in contrast to active swimming by culicine larvae used in the experiments by Matias and Adrias [1]. Even if Nothobranchius fishes were able to eradicate mosquito larvae in temporary habitats in which they are capable of surviving, their special habitat requirements prevent them from inhabiting many typical habitats of mosquito larvae, such as almost any type of receptacle capable of holding even very small amounts of rainwater (including waste items such as bottles, cans and any water containers in households) or cattle hoofprints (very typical feature of African countryside with a human settlement). These small bodies of water are significant habitats for mosquito larvae, but are impossible to stock with predators because of their abundance and small size [24]. This points to the serious logistical impracticality of the proposed scheme.

\section{Ecological threats from introductions}

Countries of sub-Saharan Africa are richly endowed with many species of Nothobranchius and related genera of annual fishes. For example, the coastal region of Tanzania alone includes more than 20 species of Nothobranchius. The assertion that $N$. guentheri is native to Tanzania [1] is correct but very imprecise, since that species is endemic to Zanzibar Island and does not occur on the African mainland [15]. As pointed out by Matias and Adrias [1], there are indeed localities with more than a single Nothobranchius species [4,14,15], but this does not diminish the potential threat of hybridization between indigenous and introduced species. There are numerous accounts of introgressive hybridization between native and introduced congeneric species in many organisms [25], including killifishes [26]. For example, hybridization between a non-native species of pupfish (Cyprinodon variegatus) and its native congener not only resulted in extinction of the native species via hybridization but also facilitated a further expansion of non-native pupfish and its hybrid swarm [27]. Within the genus Nothobranchius alone, Reichard and Polačik [28] experimentally showed that there were almost no reproductive isolating barriers between an island and 
mainland populations of $N$. korthausae despite their significant morphological and genetic differentiation leading some authors to assign the mainland populations as a separate species [29]. The risk of hybridization is especially high in taxa where sexual selection plays an important role in reproductive success [30] as is the case in Nothobranchius with brightly coloured males [31]. Native species (or populations) may show a preference for partners from non-native populations/species [32,33], which promotes rapid extinction via extensive hybridization [34].

The second potential threat to native Nothobranchius species arises from interspecific competition [25]. The ecological niche of most Nothobranchius fishes is similar $[15,16,21]$ and, at present, there is serious lack of information on how Nothobranchius communities (presence/ absence of species and their relative abundance) are shaped. Despite the fact that seemingly ecologically similar species may co-occur syntopically [14], in most habitats with multiple sympatric Nothobranchius species, the species present do appear to be ecologically separated [15].

Finally, there is an important risk of introducing diseases [35]. Introduced fish may transmit diseases contracted during captive breeding into the natural environment, with devastating consequences on natural populations [36]. Infections of serious pathogens such as the microporidian Glugea sp. are common in Nothobranchius cultures [37].

In summary, we believe that transport of Nothobranchius fish and eggs outside their current range is irresponsible and may have significant effects on the distribution of native congeners as well as other organisms in aquatic communities.

\section{Ethical issues and incorrect statements}

We believe that the Matias and Adrias initiative [1] is driven by the necessity to develop a new approach to combat infectious insect-borne diseases. The authors, both affiliated with the Poseidon Science Foundation, a non-profit branch of Poseidon Sciences biotechnological company with a large portfolio of insect (mosquito) control products [38], stated that there were no competing interests and hence we assume that the study constitutes an attempt to generously contribute to a mosquito control programme without any prospect of commercial or other interests. We welcome this attempt, but urge the authors to adhere to the mission targets of the Poseidon Sciences Foundation that include protection and preservation of the aquatic environment as their first aim [7].

We think that the recommendations given in the paper [1] pose a potentially very significant threat to natural communities in temporary freshwater pools across the African continent and adjacent islands. We are pleased that authors are aware that the introduction of $N$. guentheri to non-native areas within Tanzania and elsewhere, is inappropriate. A solution offered by Matis and Adrias is that another species with wider distribution, such as N. melanospilus, may be used instead. While this may represent a step forward from the original plans, it should be pointed out that: (1) N. melanospilus populations are variable and have a strong phylogeographical pattern, which is common to all Nothobranchius species studied to date [39], and hence even translocation within its wide range is not recommended; (2) N. melanospilus will probably hybridize with related and currently allopatric/parapatric species such as N. makondorum or N. lucius; (3) N. melanospilus may pose a risk to other native Nothobranchius via competition (including its indirect effects [25]); (4) experimental data obtained for $N$. guentheri may not be transferable to other species of the genus [40].

The study by Matias and Adrias misquotes the results of previous studies (see an example above on mosquito larvae as the dominant natural prey) and includes some incorrect statements. For example, the statement that juvenile Nothobranchius do not feed for the first three days is highly inaccurate since the juveniles must start feeding within a few hours after hatching (certainly within less than a day) in order to survive [16]. Another important fact is that the photograph published in the Matias and Adrias paper as N. guentheri, the study species, actually depicts a different Nothobranchius species (an undescribed $N$. sp. aff. kirki from Malawi), that is morphologically very different to $N$. guentheri and likely not even closely related. It is therefore unclear whether $N$. sp. aff. kirki or $N$. guentheri were used for the experiments. Also a map depicting the distribution of African annual fishes (Figure 8 in [1]) is largely incorrect, even if other African genera with potentially annual species (Fundulopanchax, Callopanchax, and Raddaella) are included.

In conclusion we reiterate that we are aware of serious problems with mosquito-borne diseases in tropical Africa and elsewhere, and we encourage responsible means of biological control of parasite vectors. There are several cases where native fish species were found to be effective predators of mosquito larvae [e.g. [41,42]] but there are also many cases where the ecological balance has been catastrophically affected [e.g. [25,43,44]]. We believe that any serious attempt to advocate particular fish species as a biocontrol agent needs to be based on sound scientific attempts to assess both the ability of species to control the parasite vector or pest, and the potential impact on natural ecosystems [23]. Otherwise, there is a significant risk of repeating mistakes made in the past decades, such as the introduction of Gambusia 
holbrooki into many countries with catastrophic consequences for native species $[43,44]$ and with no effect on mosquito populations $[45,46]$.

\author{
Acknowledgements \\ Opinions expressed in the paper are personal opinions of the authors and \\ do not represent an official view of any institution of their affiliation. The \\ paper contains a consensus of the authors' opinions on particular aspects of \\ the use of Nothobranchius in mosquito biocontrol. Financial support for \\ publication fees came from Czech Science Foundation project 206/09/0815 \\ awarded to M.R.
}

\section{Author details}

'Institute of Vertebrate Biology, Academy of Sciences of the Czech Republic, Květná 8, 60365 Brno, Czech Republic. ${ }^{2}$ Department of Geology, University of Regina, Regina, Saskatchewan S4 S OA2, Canada. ${ }^{3}$ Musée Royal de I'Afrique Centrale, 3080 Tervuren, Belgium. ${ }^{4}$ Zoologisches Forschungsmuseum Alexander Koenig, Adenauerallee 160, D-53113 Bonn, Germany. ${ }^{5} 30$, rue du Mont Ussy, 77300 Fontainebleau, France. ${ }^{6}$ Institute of Vertebrate Biology, Academy of Sciences of the Czech Republic, Květná 8, 60365 Brno, Czech Republic. 'Via Ca' Bertacchi 5, 42030 Viano (RE), Italy. ${ }^{8}$ Biology of Aging, Leibniz Institute for Age Research - Fritz Lipmann Institute, Beutenbergstr. 11, 07745 Jena, Germany \& Scuola Normale Superiore, Plazza dei Cavalieri 6, 56100 Pisa, Italy. ${ }^{9}$ Professor Emeritus, College of Veterinary Medicine, Cornell University, Ithaca, NY 14853, USA.

${ }^{10}$ Tegernseerlandstraße 42, 81541 Munich, Germany. ${ }^{11}$ Scandinavian Killifish Association, Copenhagen, Denmark. ${ }^{12} 22$ Horton Road, Slapton, Leighton Buzzard, Beds., LU7 9DB, UK.

\section{Authors' contributions}

MR, BRW, RHW initiated the report. MR drafted the manuscript. All authors contributed to and approved the final version of the manuscript.

\section{Authors' information}

The authors include active researchers on African killifish ecology and evolution and specialized Nothobranchius fish keepers, members of several national killifish keeping societies. The authors regularly collect Nothobranchius fishes in their natural habitats.

\section{Competing interests}

The authors declare no financial, academic or intellectual competing interests. We acknowledge that our opinions are based on our personal views on how the relationship between environmental protection and development should be assessed.

Received: 9 September 2010 Accepted: 16 September 2010 Published: 16 September 2010

\section{References}

1. Matias JR, Adrias AQ: The use of annual killifish in the biocontrol of the aquatic stages of mosquitoes in temporary bodies of fresh water; a potential new tool in vector control. Parasites \& Vectors 2010, 3:46.

2. Vanderplank FL: Nothobranchius and Barbus species: indigenous antimalaria fishes in East Africa. East Africa Med J 1941, 17:431-436.

3. Myers GS: Annual Fishes. Aquar J 1952, 22:145-151.

4. Wildekamp RH: Assignment report. Preliminary study of the Somalian Nothobranchius species of larvivorous fishes. EM/MAL/198 EMNBC/41 SOM/MPD/001/RB World Health Organization 1983.

5. Markofsky J, Matias JR: Waterborne vectors of disease in tropical and subtropical areas and novel approach to mosquito control using annual fish. The Columbia University Seminars on Pollution and Water Resource 1979, 12:H1-H17.

6. Haas R: Assignment report. Malaria control programme. EMNBC/18, EM/ MAL/164 World Health Organization 1979.

7. Matias JR: Poseidon Science Foundation and the government of Tanzania explore a vector control method using native larvivorous annual fish that mimic life cycle of malarial mosquitoes. 2009 [http:// www.poseidonsciences.com/Malaria_control_mosquito_larvivorous_annual_ fish_Nothobranchius_temporary_habitats_TPRI_Poseidon_Tanzania.pdf].
8. Watters BR: The ecology and distribution of Nothobranchius fishes. J Am Killifish Assoc 2009, 42:58-61.

9. Watters BR: Dispersal of Nothobranchius - Fact and Fiction. J Am Killifish Assoc 2006, 39:137-144.

10. Watters BR: Report on the investigation of Nothobranchius fish localities in the Kruger National Park, 1999-2003. Skukuza: South African National Parks 2003.

11. Watters BR: A study of Nothobranchius habitats in the Great Limpopo Transfrontier Park. J Am Killifish Assoc 2006, 39:14-25.

12. Vanderplank FR: Why not "instant" fish farms? New Scientist 1967, 33:42.

13. Wildekamp RH: Redescription of two lesser known Nothobranchius from Central Tanzania, N. taeniopygus and N. neumanni (Cyprinodontiformes: Aplocheilidae). Ichthyol Explor Fres 1990, 1:16.

14. Reichard M, Polačik M, Sedláček O: Distribution, colour polymorphism and habitat use of the African killifish, Nothobranchius furzeri, the vertebrate with the shortest lifespan. J Fish Biol 2009, 74:198-212.

15. Wildekamp RH: A World of Killies: Atlas of the Oviparous Cyprinodontiform Fishes of the World. Elyria: American Killifish Association 2004, 4.

16. Neumann W: Prachtgrundkärpflinge. Supplementheft Nr. 9. Zwickau: Deutsche Killifisch Gemeinschaft 2008.

17. Matias JR: The stage-dependent resistance of the chorion to external chemical damage and its relationship to embryonic diapause in the annual fish, Nothobranchius guentheri. Experientia 1984, 40:753-754.

18. Kovchazov G: Assignment report. Local larvivorous fish for mosquito control. Malaria pre-eradication programme, Somalia. EM/MAL/128, Somalia 2001R, World Health Organization 1974.

19. Djelantik AAM: Assignment report. Malaria pre-eradication programme, Somalia (SOM/68/016). EM/MAL 137, EM/SOM/MPD001/RB/DP 92001, World Health Organization 1975.

20. Wildekamp RH, Haas R: Redescription of Nothobranchius microlepis, description of two new species from northern Kenya and southern Somalia, and note on the status of Paranothobranchius

(Cyprinodontiformes: Aplocheilidae). Ichthyol Explor Fres 1992, 3:1-16.

21. Polačik M, Reichard M: Diet overlap among three sympatric African annual killifish species (Nothobranchius spp.) from Mozambique. J Fish Biol 2010, 77:754-768.

22. Wellborn GA, Skelly DK, Werner EE: Mechanisms creating community structure across a freshwater habitat gradient. Annu Rev Ecol Sys 1996, 27:337-363.

23. Louca V, Lucas MC, Green C, Majambere S, Fillinger U, Lindsay SW: Role of fish as predators of mosquito larva on the floodplain of the Gambia River. J Medical Entomol 2009, 46:546-556.

24. Bahar R: Report on a visit to Somalia, 15-30 January 1974. EM/MAL124, SOM 2001/R, UNDPdd, World Health Organization 1974.

25. Mooney HA, Cleland EE: The evolutionary impact of invasive species. Proc Natl Acad Sci USA 2001, 98:5446-5451.

26. Kodric-Brown A, Rosenfield JA: Populations of Pecos pupfish (C. pecosensis) differ in their susceptibility to hybridization with sheepshead minnow (C. variegatus). Behav Ecol Sociobiol 2004, 56:116-123.

27. Rosenfield JA, Nolasco S, Lindauer S, Sandoval C, Kodric-Brown A: The role of hybrid vigor in the replacement of Pecos pupfish by its hybrids with sheepshead minnow. Conserv Biol 2004, 18:1-10.

28. Reichard M, Polačik M: Reproductive isolating barriers between colourdifferentiated populations of an African annual killifish, Nothobranchius korthausae (Cyprinodontiformes). Biol J Linn Soc 2010, 100:62-72.

29. Costa WJEM: Species delimitation among populations of the eastern Tanzanian seasonal killifish Nothobranchius korthausae (Cyprinodontiformes: Nothobranchiidae). Ichthyol Explor Fres 2009, 20:111-126.

30. Kodric-Brown A, Strecker U: Responses of Cyprinodon maya and C. labiosus females to visual and olfactory cues of conspecific and heterospecific males. Biol J Linn Soc 2001, 73:541-548.

31. Haas R: Sexual selection in Nothobranchius guentheri (Pisces: Cyprinidontidae). Evolution 1976, 30:614-622.

32. Ryan MJ, Wagner WE Jr: Asymmetries in mating preferences between species: female swordtails prefer heterospecific males. Science 1987, 236:595-597.

33. Wong BBM, Fisher HS, Rosenthal GG: Species recognition by male swordtails via chemical cues. Behav Ecol 2005, 16:818-822. 
34. Rosenfield J, Kodric-Brown A: Sexual selection promotes hybridization between Pecos pupfish, Cyprinodon pecosensis-C. variegatus and sheepshead minnow. J Evol Biol 2003, 16:595-606.

35. Krkosek M, Ford JS, Morton A, Lele S, Myers RA, Lewis MA: Declining wild salmon populations in relation to parasites from farm salmon. Science 2007, 318:1772-1775.

36. Daszak P, Cunningham AA, Hyatt AD: Emerging infectious diseases of wildlife - threats to biodiversity and human health. Science 2000, 287:443-449.

37. Lom J, Noga EJ, Dyková I: Occurrence of a microsporean with characteristics of Glugea anomala in ornamental fish of the family Cyprinodontidae. Dis Aquat Org 1995, 21:239-242.

38. Poseidon Ocean Sciences, Inc: Poseidon Sciences.[http://www. poseidonsciences.com/].

39. Terzibasi E, Valenzano DR, Benedetti M, Roncaglia P, Cattaneo A, Domenici L, Cellerino A: Large differences in aging phenotype between strains of the short-lived annual fish Nothobranchius furzeri. PLOS ONE 2008, 3:e3866.

40. Laufer G, Arim M, Loureiro M, Pineiro-Guerra JM, Clavijo-Baquet S, Fagundez C: Diet of four annual killifishes: an intra and interspecific comparison. Neotrop Ichthyol 2009, 7:77-86.

41. Howard AFV, Zhou G, Omlin FX: Malaria mosquito control using edible fish in western Kenya: preliminary findings of a controlled study. BMC Public Health 2007, 7:199.

42. Ghosh SK, Tiwari SN, Sathyanarayan TS, Sampath TRR, Sharma VP, Nanda N, Joshi H, Adak T, Subbarao SK: Larvivorous fish in wells target the malaria vector sibling species of Anopheles culicifacies complex in villages in Karnataka, India. Trans Royal Trop Med Hygiene 2005, 99:101-105.

43. García-Berthou E, Alcaraz C, Pou-Rovira Q, Zamora L, Coenders G, Feo C: Introduction pathways and establishment rates of invasive aquatic species in Europe. Can J Fish Aquat Sci 2005, 62:453-463.

44. Leyse KE, Lawler SP, Strange T: Effects of an alien fish, Gambusia affinis, on an endemic California fairy shrimp, Linderiella occidentalis: implications for conservation of diversity in fishless waters. Biol Conserv 2004, 118:57-65.

45. Willems $K$, Webb CE, Russell RC: A comparison of mosquito predation by the fish Pseudomugil signifier Kner and Gambusia holbrooki (Girard) in laboratory trials. J Vector Ecol 2005, 30:87-90.

46. Blaustein L: Larvivorous fishes fail to control mosquitoes in experimental rice plots. Hydrobiologia 1992, 232:219-232.

doi:10.1186/1756-3305-3-89

Cite this article as: Reichard et al:: Potential negative impacts and low effectiveness in the use of African annual killifish in the biocontrol of aquatic mosquito larvae in temporary water bodies. Parasites \& Vectors 2010 3:89.

\section{Submit your next manuscript to BioMed Central and take full advantage of:}

- Convenient online submission

- Thorough peer review

- No space constraints or color figure charges

- Immediate publication on acceptance

- Inclusion in PubMed, CAS, Scopus and Google Scholar

- Research which is freely available for redistribution 